Case Report

\title{
Upper Limb Deep Vein Thrombosis in Patient with Hemophilia A and Heterozygosity for Prothrombin G20210A: A Case Report and Review of the Literature
}

\author{
Fares Darawshy, ${ }^{1}$ Yosef Kalish, ${ }^{2}$ Issam Hendi, ${ }^{1}$ Ayman Abu Rmelieh, ${ }^{1}$ and Tawfik Khoury ${ }^{1}$ \\ ${ }^{1}$ Department of Medicine, Hadassah-Hebrew University Medical Center, Jerusalem, Israel \\ ${ }^{2}$ Department of Hematology, Hadassah-Hebrew University Medical Center, Jerusalem, Israel \\ Correspondence should be addressed to Tawfik Khoury; tawfikkhouryl@hotmail.com
}

Received 27 April 2017; Revised 12 August 2017; Accepted 22 August 2017; Published 25 September 2017

Academic Editor: Simon Davidson

Copyright (c) 2017 Fares Darawshy et al. This is an open access article distributed under the Creative Commons Attribution License, which permits unrestricted use, distribution, and reproduction in any medium, provided the original work is properly cited.

Deep vein thrombosis (DVT) is a rare disease in patients with hemophilia A. We report a case of 22-year-old male with severe hemophilia A who presented to the emergency room with 5-day history of right arm pain that was attributed initially to bleeding event. In the absence of external signs of bleeding or hematoma and normal hemoglobin level, we suspected an underlying DVT. Doppler ultrasonography of the right upper limb revealed thrombosis of the subclavian vein and this was confirmed by CT venography. The d-dimer level was normal and investigations for prothrombotic state revealed heterozygosity for prothrombin G20210A mutation. Treatment with factor VIII and low molecular weight heparin led to successful resolution and marked improvement of his clinical condition.

\section{Introduction}

Hemophilia A is a hereditary hemorrhagic disease characterized by the deficiency of the coagulation FVIII. Due to the bleeding tendencies in hemophilia A patients, the occurrence of spontaneous venous thromboembolism is a rare and even surprising but has been reported in the literature [1-4]. The mechanism of spontaneous venous thromboembolism in hemophilia A patient is unclear due to limited number of cases and there are no clear guidelines for its treatment.

We report a case of 22-year-old male patient with severe hemophilia A who developed DVT in the right upper arm.

\section{Case Presentation}

A 22-year-old male diagnosed with severe hemophilia A on F VIII prophylaxis (recombinant F VIII therapy, 3000 units 3 times a week) self-administered into a peripheral vein of the right arm presented to the emergency room for evaluation of right arm and shoulder pain in the past 5 days. On admission, the patient was hemodynamic and respiratory stable. His physical examination was unremarkable except for right arm tenderness without swelling, erythema, or increased vascular markings.

Prothrombotic workups including protein $\mathrm{C}$ activity, protein S activity, antithrombin III level, APC resistance, lupus anticoagulant, anticardiolipin antibodies, factor VIII inhibitor screening, and factor $\mathrm{V}$ Leiden defect were all negative, except for heterozygous mutation for prothrombin G20210A; the d-dimer level was normal $0.14 \mathrm{mcg} / \mathrm{mL}(0-0.5)$ and coagulation tests revealed low FVIII levels. A Doppler ultrasonography (US) of the right upper limb showed an echogenic thrombosis in the right subclavian vein. CT venography confirmed the diagnosis of right subclavian DVT (Figure 1). All imaging studies ruled out thoracic outlet syndrome.

Further investigations were performed to rule out other causes of DVT; inflammatory markers $\mathrm{C}$ reactive protein (CRP) and erythrocyte sedimentation rate (ESR) were within normal range (Table 1). HLA-B51 was performed to rule out Bechet disease and was negative.

The patient was treated with low molecular weight heparin (LMWH) enoxaparin $100 \mathrm{mg}$ once daily $(1.5 \mathrm{mg} / \mathrm{kg})$, 

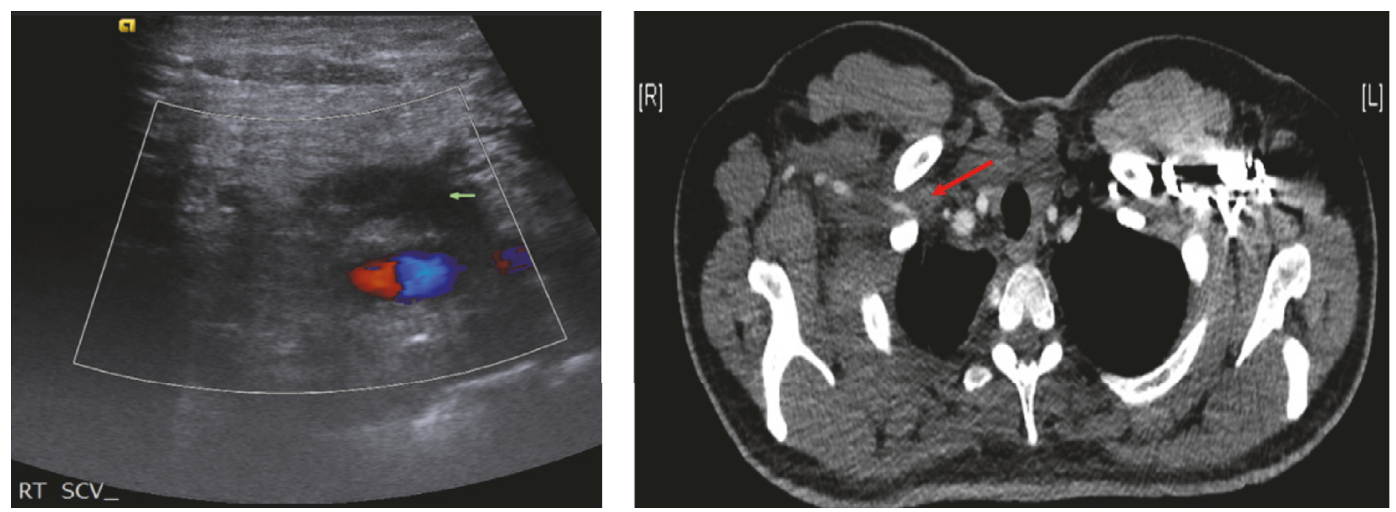

FIgURE 1: Doppler US and CT scan showing DVT in the right subclavian vein. Arrows refer to deep vein thrombosis.

TABLE 1: Laboratory tests results.

\begin{tabular}{|c|c|c|}
\hline Parameter & Result & Normal values \\
\hline WBC count $\left(* 10^{9} / \mathrm{L}\right)$ & 8.4 & $4-10$ \\
\hline Platelets count $\left(* 10^{9} / \mathrm{L}\right)$ & 209 & $140-400$ \\
\hline Hemoglobin (Gr\%) & 15.1 & $14-18$ \\
\hline PT (\%) & 73.03 & $60-100$ \\
\hline aPTT (s) & 54.7 & $25-49$ \\
\hline INR & 1.22 & $1-1.4$ \\
\hline D-DIMER (mcg/mL) & 0.14 & $0-0.5$ \\
\hline Factor VIII level (\%) & 17 & $70-140$ \\
\hline Factor VIII inhibitor screening & Negative & \\
\hline Protein $\mathrm{C}$ activity & Normal & $70-120$ \\
\hline Protein $S$ activity & Normal & $70-140$ \\
\hline Antithrombin III level & Normal & \\
\hline APC resistance & Negative & \\
\hline Lupus anticoagulant & Absent & \\
\hline Anticardiolipin antibodies & Absent & \\
\hline Factor V Leiden defect & Absent & \\
\hline Prothrombin G20210A & Heterozygote & \\
\hline ESR & 28 & $1-20$ \\
\hline CRP (mg\%) & 1.42 & $<0.5$ \\
\hline ANA & Absent & \\
\hline C-ANCA (unit/mL) & 8 & $>18$ \\
\hline P-ANCA (unit/mL) & 1.6 & $>18$ \\
\hline C3 (mg/dL) & 138 & $90-180$ \\
\hline $\mathrm{C} 4(\mathrm{mg} / \mathrm{dL})$ & 27 & $10-40$ \\
\hline
\end{tabular}

along with prophylactic FVIII 2000 units per day. This regimen increased FVIII levels up to $49.2 \%$ measured 1 hour after FVIII injection and was continued for 2 months.

After two months, the dose of LMWH was reduced to $40 \mathrm{mg}$ and the dose of FVIII was escalated to 3000 units, given three times a week for another 3 months, followed by another six weeks of treatment with $40 \mathrm{mg}$ LMWH and 2000 units of FVIII administered every other day. There was complete resolution of DVT accompanied with significant clinical improvement without recurrence of the thrombosis to date.

\section{Discussion}

Thrombotic events have been rarely reported among patients with hemophilia [1-3]. Goodnough et al. [5] found no thrombotic complications in 178 patients with hemophilia A over a 30-year follow-up period. The exact pathomechanism and the predisposing factors for the occurrence of venous thrombosis in hemophilic patients are still not well established. Kasper found no evidence of thrombosis in major orthopedic operations in patients with hemophilia A [6]. However, Pruthi et al. reported case report of DVT in hemophilia B patient and factor $\mathrm{V}$ Leiden following hip 
fracture surgery [7]. In another recent study, Buckner et al. reported symptomatic venous thromboembolism incidence of $4.3 \%$ in hemophilia patients undergoing major orthopedic surgery [8]. Girolami et al. have reviewed all reported patients with hemophilia A and hemophilia B in the literature and found that the administration of factor VIII inhibitor bypassing activity (FEIBA) or recombinant activated FVII (rFVIIa) in patients with inhibitors and surgery was the most frequent risk factor for thromboembolism development in hemophilia A and B patients, respectively, in addition to variable prothrombotic conditions and administration of prothrombin complex concentrate (PCC) [9]. Stewart et al. reported the occurrence of venous thrombosis in a patient with hemophilia A after a long flight [4]. On the other hand, few previous case reports reported venous thrombosis in hemophilia A with no prothrombotic risk factor identified $[2,3]$. Our patient developed right subclavian DVT in the arm used for self-injected FVIII prophylactic therapy and was found to have heterozygous mutation for prothrombin G20210A. There were no other identifiable predisposing risk factors as assessed by an extensive investigation that ruled out inflammatory, mechanical obstructive, and neoplastic causes. Prothrombin is a precursor of the serine protease thrombin and is a key enzyme in the process of hemostasis. Prothrombin heterozygosity is related to a single-nucleotide substitution ( $\mathrm{G}$ to $\mathrm{A}$ ) at position 20210 in the $3^{\prime}$ untranslated region of the gene encoding prothrombin. Its heterozygous state, 20210A, is a risk factor for the development of deep vein thrombosis [10]. Deep vein thrombosis and cerebral vein thrombosis have been reported in association with heterozygous prothrombin mutation $[11,12]$. Interestingly, one may postulate that the low FVIII in our patient of $17 \%$ is in favor of bleeding tendency; however, the occurrence of DVT in the setting of low systemic level of FVIII may suggest the strong hypercoagulability potential of heterozygous prothrombin G20210A mutation or, on the other hand, might suggest the hypothesis of FVIII administration as a predisposing factor for local thromboembolism development in hemophilia A patients. Another interesting point is that our patient had normal d-dimer level although he had upper extremity DVT. Normal d-dimer level in two patients with pacemaker and upper extremity DVT was previously reported [13]. In a study for evaluating the usefulness of d-dimer in the evaluation of upper extremity DVT [14], the reported sensitivity was 100\% but the $95 \%$ confidence interval was $78-100$. The specificity was $14 \%$, not surprising given the poor specificity of ddimer in venous thromboembolism. Based on these previous studies, the use of d-dimer in our case to rule out upper extremity DVT is limited and a Doppler US should be the initial test of choice for upper extremity DVT once suspected.

The treatment of spontaneous venous thrombosis in patients with hemophilia $\mathrm{A}$ is not well defined, and the recommended treatment of this condition is based on case reports as different regimens have been used for its treatment. Stewart et al. [4] treated their patient with LMWH for 5 days and tinzaparin for 6 weeks. Dargaud et al. used the unfractionated heparin in addition to FVIII replacement therapy for 1 month [2]. Kashyap et al. used LMWH for 9 weeks [3]. Oral anticoagulation was not used, probably since it increases the risk of bleeding. Bicer et al. [1] treated their patient with LMWH for 2 days followed by warfarin for 6 weeks. No bleeding complications were reported in all cases.

In our case, we treated the patient according to hematological consultation with LMWH on one hand for DVT and with FVIII prophylaxis treating the hemophilia A coagulopathy on the other hand. The regimen was continued for 3 months and then tapered down the administration of LMWH for another six weeks till treatment cessation. This patient was monitored closely and marked clinical and radiological improvement was observed.

In conclusion, spontaneous venous thromboembolism is a very rare and unexpected disease in patients with hemophilia A. Clinicians should be aware about this entity and should consider this differential diagnosis per patient individually especially in the FVIII injected limb. This case shed light on the uncertainty of the mechanism involved in the occurrence of venous thromboembolism in hemophilia A patients especially with low FVIII level. Therefore, further preclinical and clinical trials should be carried out to elucidate the mechanism and treatment of venous thrombosis in patients with hemophilia and to perform larger studies evaluating the exact predictive value of d-dimer in upper limb DVT.

\section{Conflicts of Interest}

The authors report no conflicts of interest regarding this manuscript.

\section{Authors' Contributions}

Fares Darawshy, Tawfik Khoury, and Yosef Kalish contributed to the concept and design of the study, all authors contributed to the analysis and interpretation of data, Fares Darawshy and Tawfik Khoury contributed to critical writing and revising the intellectual content, and all authors approved the final version to be published.

\section{References}

[1] M. Bicer, M. Yanar, and O. Tuydes, "Spontaneous deep vein thrombosis in hemophilia A: a case report," Cases Journal, vol. 2, no. 9, Article no. 6390, 2009.

[2] Y. Dargaud, B. B. Cruchaudet, A. Lienhart, B. Coppéré, J. Ninet, and C. Négrier, "Spontaneous proximal deep vein thrombosis in a patient with severe haemophilia A," Blood Coagulation and Fibrinolysis, vol. 14, no. 4, pp. 407-409, 2003.

[3] R. Kashyap, L. M. Sharma, S. Gupta, R. Saxena, and D. N. Srivastava, "Deep vein thrombosis in a patient with severe haemophilia A," Haemophilia, vol. 12, no. 1, pp. 87-89, 2006.

[4] A. J. Stewart, L. M. Manson, R. Dennis, P. L. Allan, and C. A. Ludlam, "Thrombosis in a duplicated superficial femoral vein in a patient with haemophilia A," Haemophilia, vol. 6, no. 1, pp. 47-49, 2000.

[5] L. T. Goodnough, H. Saito, and O. D. Ratnoff, “Thrombosis or myocardial infarction in congenital clotting factor abnormalities and chronic thrombocytopenias: A report of 21 patients and 
a review of 50 previously reported cases," Medicine, vol. 62, no. 4, pp. 248-255, 1983.

[6] C. K. Kasper, "Postoperative thromboses in hemophilia B," New England Journal of Medicine, vol. 289, no. 3, 160 pages, 1973.

[7] R. K. Pruthi, J. A. Heit, M. M. Green et al., "Venous thromboembolism after hip fracture surgery in a patient with haemophilia B and factor V Arg506Gln (factor V Leiden)," Haemophilia, vol. 6, no. 6, pp. 631-634, 2000.

[8] T. W. Buckner, A. D. Leavitt, M. Ragni et al., "Prospective, multicenter study of postoperative deep-vein thrombosis in patients with haemophilia undergoing major orthopaedic surgery," Thrombosis and Haemostasis, vol. 116, no. 1, pp. 42-49, 2016.

[9] A. Girolami, R. Scandellari, E. Zanon, R. Sartori, and B. Girolami, "Non-catheter associated venous thrombosis in hemophilia A and B. A critical review of all reported cases," Journal of Thrombosis and Thrombolysis, vol. 21, no. 3, pp. 279-284, 2006.

[10] M. W. Kellett, P. J. Martin, T. P. Enevoldson, C. Brammer, and C. M. Toh, "Cerebral venous sinus thrombosis associated with 20210A mutation of the prothrombin gene," Journal of Neurology Neurosurgery and Psychiatry, vol. 65, no. 4, pp. 611612, 1998.

[11] V. Biousse, J. Conard, C. Brouzes, M. H. Horellou, and A. Ameri, "Frequency of the $20210 \mathrm{G}->$ A mutation in the 3'-untranslated region of the prothrombin gene in 35 cases of cerebral venous thrombosis," Stroke, vol. 29, pp. 1398-1400, 1998.

[12] O. Hudaoglu, S. Kurul, U. Yis, E. Dirik, H. Cakmakci, and S. Men, "Basilar artery thrombosis in a child heterozygous for prothrombin gene G20210A mutation," in Journal of Child Neurology, vol. 22, pp. 329-331, 22, 2007.

[13] C. Byrne, J. Abdulla, and J. K. Christensen, "Normal D-dimer in two patients with pacemaker and deep venous thrombosis in an upper extremity," in Ugeskr Laeger, vol. 177, pp. 12-13, 2015.

[14] T. Merminod, S. Pellicciotta, and H. Bounameaux, "Limited usefulness of D-dimer in suspected deep vein thrombosis of the upper extremities," Blood Coagulation and Fibrinolysis, vol. 17, no. 3, pp. 225-226, 2006. 


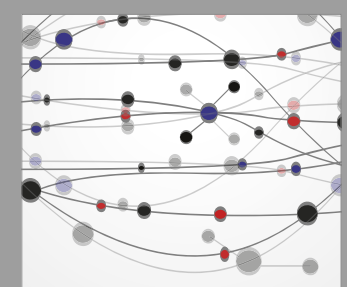

The Scientific World Journal
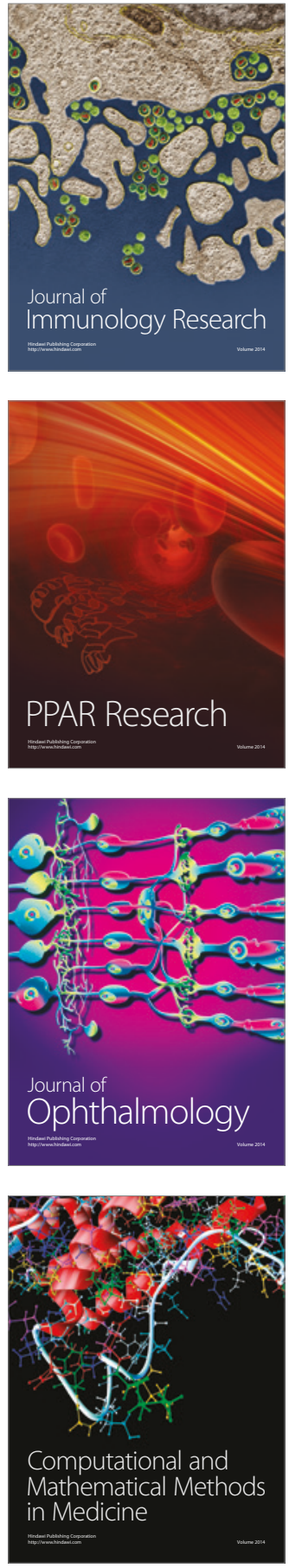

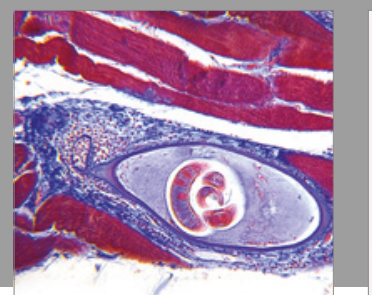

Gastroenterology Research and Practice
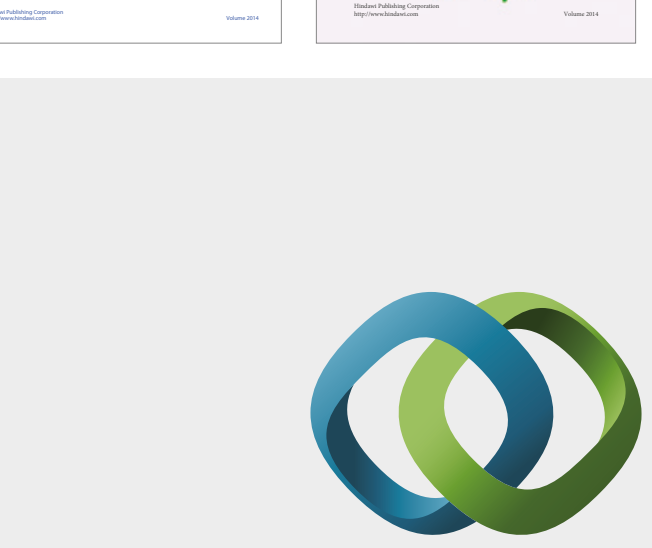

\section{Hindawi}

Submit your manuscripts at

https://www.hindawi.com
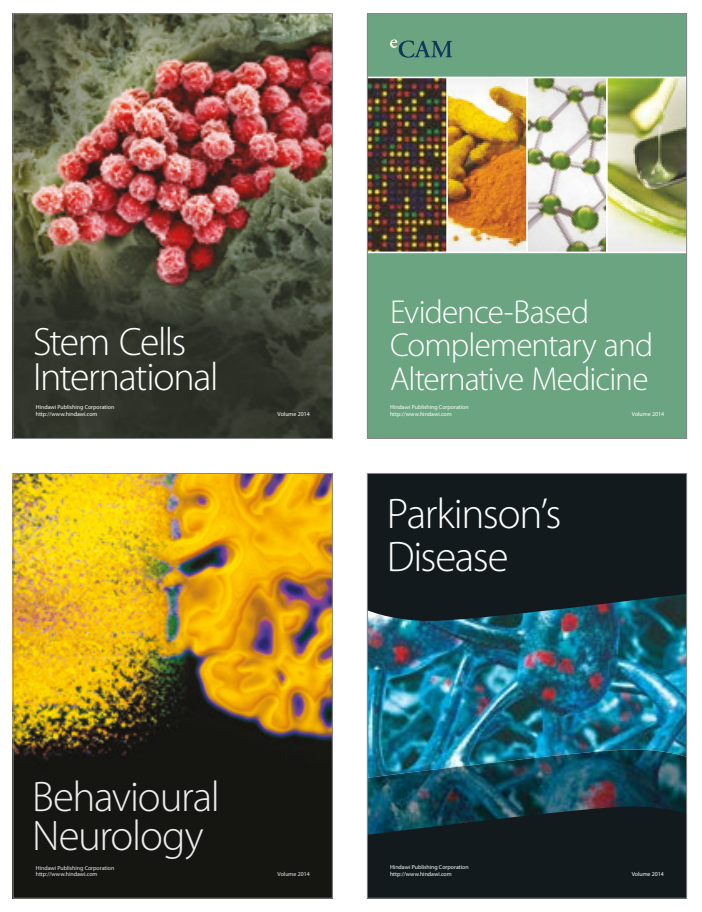
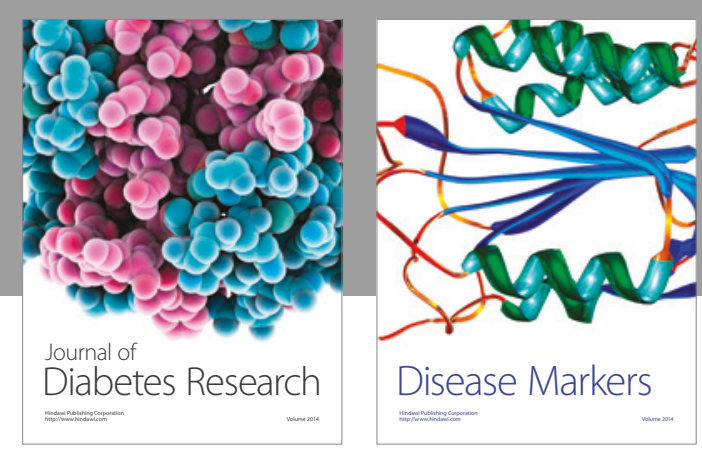

Disease Markers
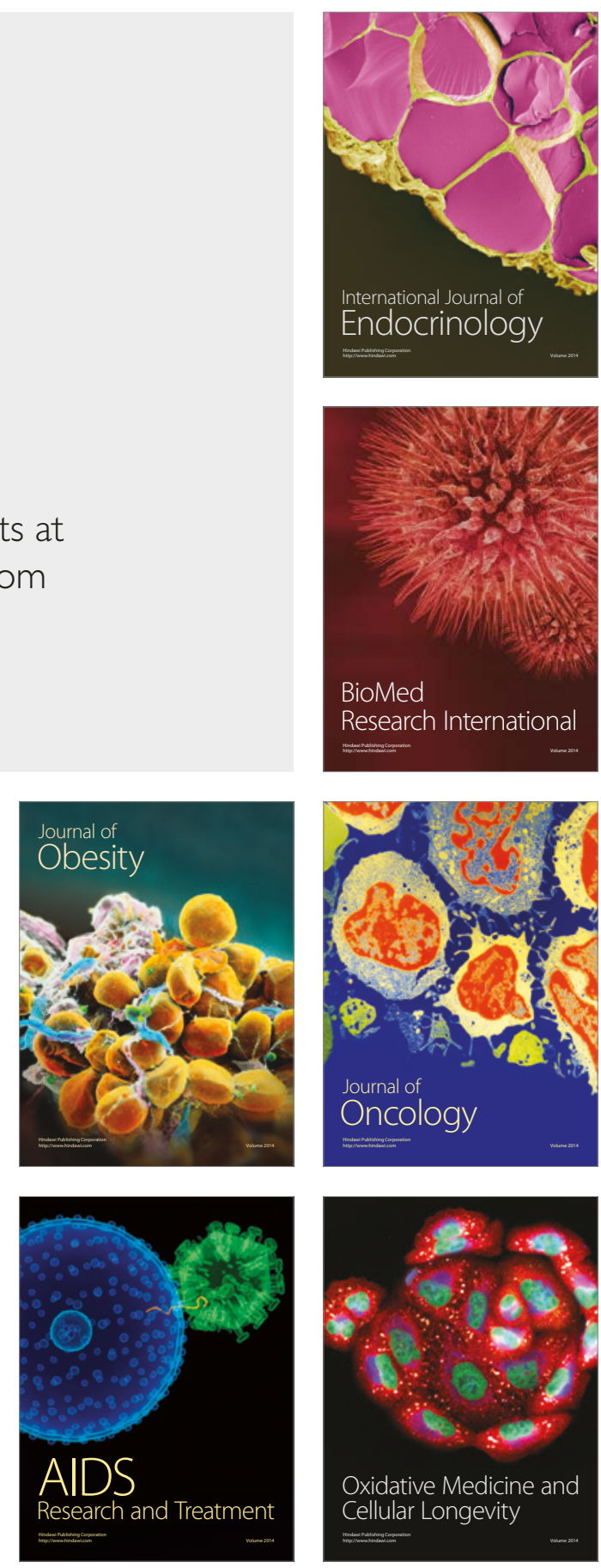